\title{
Ultrasonography-Based Management of Sclerosing Mesenteritis: From Diagnosis to Follow-Up
}

This article was published in the following Dove Press journal:

International Medical Case Reports Journal

\author{
Roberto de Sire' \\ Nicola Imperatore ${ }^{1,2}$ \\ Maria D'Armiento $\mathrm{Snr}^{3}$ \\ Pietro Coccoli' \\ Imma Di Luna' \\ Simona Ricciolino' \\ Fabiana Castiglione' \\ Antonio Rispo' \\ 'Gastroenterology, Department of \\ Clinical Medicine and Surgery, University \\ Federico II of Naples, Naples, Italy; \\ ${ }^{2}$ Gastroenterology and Endoscopy Unit, \\ AORN Antonio Cardarelli, Naples, Italy; \\ ${ }^{3}$ Pathology, Department of Public Health, \\ University Federico II of Naples, Naples, \\ Italy
}

\begin{abstract}
Sclerosing mesenteritis (SM) is an idiopathic disorder affecting mesentery, characterized by fat necrosis, chronic inflammation and fibrosis. The clinical presentation varies from asymptomatic cases to acute abdomen. The diagnosis is suggested by imaging but can be definitely established only by biopsies. In this paper, we discuss ultrasonographybased management of SM.
\end{abstract}

Keywords: sclerosing mesenteritis, mesenteric lipodystrophy, mesenteric panniculitis, retractile mesenteritis, ultrasonography

\section{Introduction}

Sclerosing mesenteritis (SM) is a rare, idiopathic, benign disease affecting typically the small bowel mesentery, and is characterized by fat necrosis, non-specific chronic inflammation and fibrosis. ${ }^{1}$ It was recognized for the first time by Jura in 1924 and defined as "retractile mesenteritis"; 2 over the years, several terms have been used to describe this disease, such as "mesenteric lipodystrophy", "mesenteric panniculitis", 4 "xanthogranulomatous mesenteritis" fibrosis". ${ }^{6}$ Finally, in 1997, Emory et al concluded that these different conditions represented the histologic variants of a single entity, named "sclerosing mesenteritis", the preferred term for referring to the entire disease spectrum. ${ }^{1}$

$\mathrm{SM}$ is an uncommon disorder, typically diagnosed in the fifth to seventh decade of life with an estimated prevalence of $<1 \%$ and male preponderance. ${ }^{7,8}$ Although the etiopathogenesis is still unclear, previous abdominal trauma or surgery, cancer, autoimmunity, infection, and medications have been linked to the development of SM. ${ }^{9}$

Clinical manifestation of SM could vary from asymptomatic cases, diagnosed incidentally on imaging, ${ }^{7}$ to bowel obstruction, mesenteric ischemia, chylous ascites and protein-losing enteropathy, caused by mass effect on neighboring structures. ${ }^{10-12}$ Patients commonly show abdominal pain, diarrhea, weight loss, palpable abdominal masses, abdominal tenderness, nausea, vomiting, anorexia, and fever. ${ }^{8}$ Taking into account its non-specific manifestations, radiologic imaging plays a key role in the diagnosis of SM; ${ }^{13}$ nevertheless, a definite diagnosis can be established only by surgical or imaging-guided biopsies. ${ }^{11}$

In the absence of clinical trials, the treatment of SM is empirical and ranges, according to clinical presentation, from simple observation to corticosteroids, immunosuppressive drugs, hormonal therapy or surgery. ${ }^{9}$ The progression is often benign and self-limiting with a good long-term prognosis, although some cases of severe/fatal SM have been reported in literature. ${ }^{10,11}$
Correspondence: Roberto de Sire Gastroenterology Unit, Department of Clinical Medicine and Surgery, University "Federico II" of Naples, Via Pansini 5 , Naples, 80I3I, Italy

Email roberto.desire@libero.it 
In this paper, we report an ultrasonography-based management model of SM, aimed at providing a possible diagnostic-therapeutic approach that might be useful for gastroenterology research field.

\section{Case Report}

In 2018, a 63-year-old male (height $1.81 \mathrm{~m}$, weight $63.5 \mathrm{~kg}$ ) was admitted to our hospital with a recent history of diffuse abdominal pain associated with change in bowel habits (4 bowel movements/day without mucus and blood) and severe weight loss (about $25 \mathrm{~kg}$ in the last two years). In anamnesis, he reported only classic infectious diseases of childhood. He did not refer previous surgery or familiar history of gastrointestinal diseases and cancer. Physical exam revealed a palpable abdominal mass in mesogastrium. Laboratory findings were normal in absence of anemia or leukocytosis. No serological inflammatory status was observed. Ileocolonoscopy was normal. Ultrasound (US) showed a well-defined hyperechoic mass (measuring $41 \times 110 \mathrm{~mm}$ ) in the root of the small bowel mesentery with reactive sub-centimetric node in the context of the thickened mesenteric fat (shown in Figure 1). On the basis of clinical and ultrasonographical signs, the diagnosis of "sclerosing mesenteritis" was made. After the exclusion of a possible associated immuno-mediated disorders by serology, a whole-body computed tomography (CT) scan was performed to rule out the occurrence of a concomitant neoplasia. The CT confirmed the US diagnosis of sclerosing mesenteritis by highlighting the presence of a "misty mesentery", characterized by the increase of mesenteric fat density associated with enlarged inflammatory nodes. Finally, the patient underwent a USguided biopsy of mesentery with a histology indicative for SM (shown in Figure 2). After a brief course of steroids (prednisone $50 \mathrm{mg}$ ), we decided to maintain treatment with

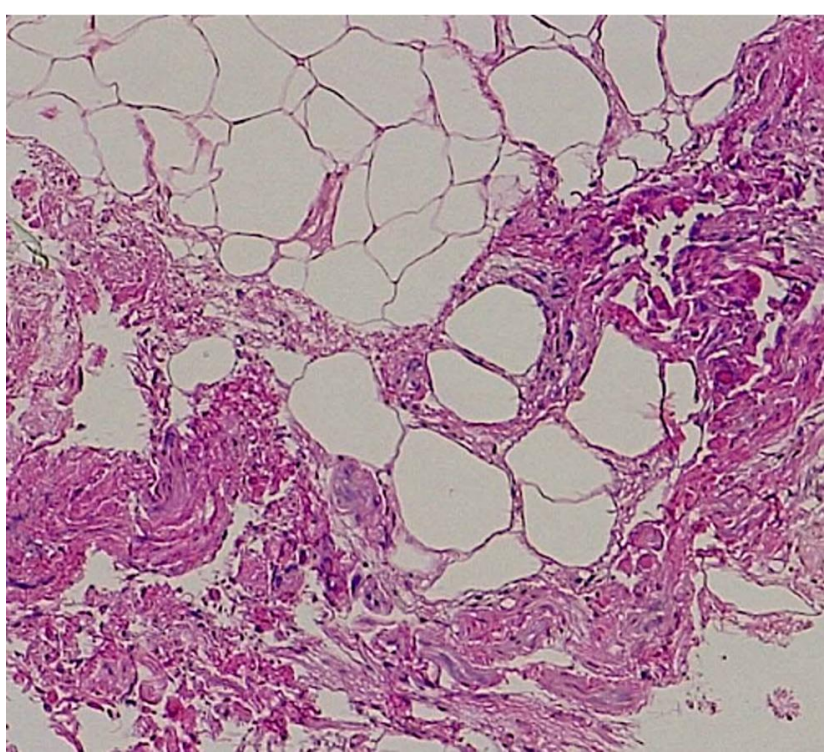

Figure 2 Sclerosing mesenteritis at histology. Fibrosis with dense collagen, fat necrosis, and chronic inflammation are evident.

tamoxifen $20 \mathrm{mg} /$ daily associated with aspirin $100 \mathrm{mg} /$ daily, in order to prevent a potential vascular complication, such as mesenteric ischemia. At US-based 6-months and 12-months follow-up visits, we recorded an improvement of US picture associated with weight gain (about $10 \mathrm{~kg}$ ) and a stable remission of diarrhea and abdominal pain. After 2 years of followup, the patient is healthy and continues to report for US-based follow-ups. No additional CT scans were needed.

\section{Discussion and Conclusion}

SM is a rare inflammatory disorder of unknown etiopathogenesis, characterized by fat necrosis, non-specific chronic inflammation and fibrosis of the small bowel mesentery. ${ }^{1}$ On the basis of predominant histology, it is possible to

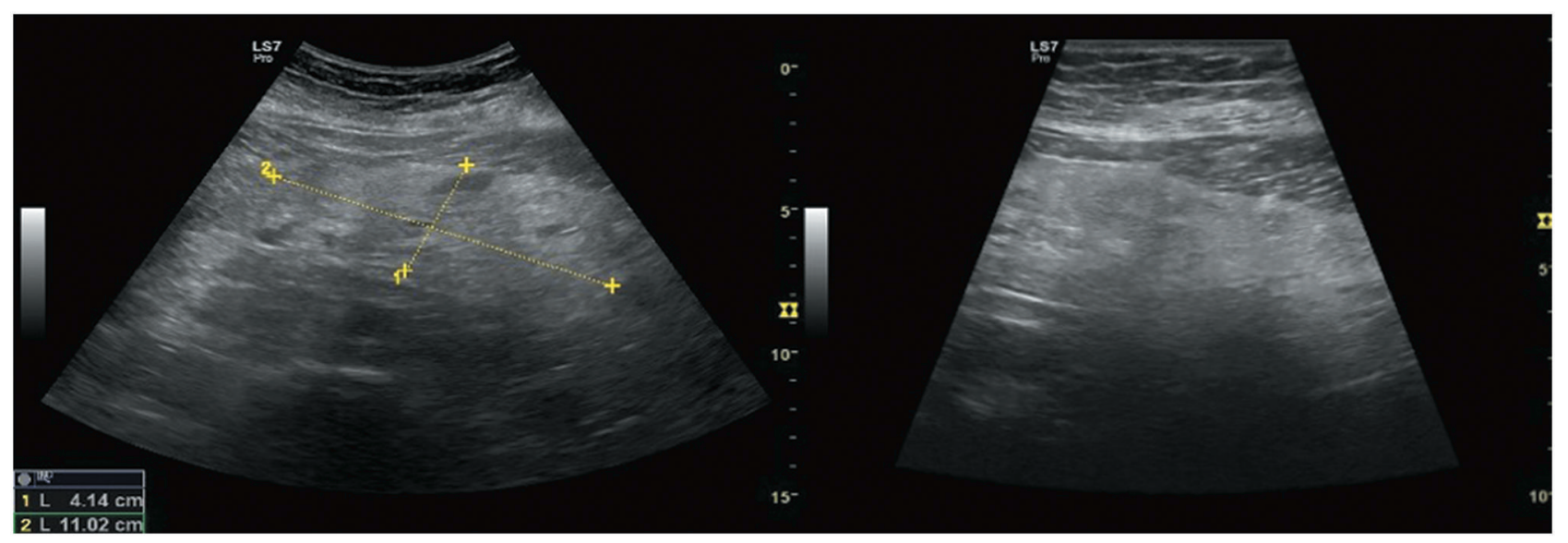

Figure I Sclerosing mesenteritis at ultrasonography. The thickened and hyperechoic mesentery is well evident. 
divide SM into three categories: mesenteric lipodystrophy (fat necrosis), mesenteric panniculitis (chronic inflammation) and retractile mesenteritis (fibrosis). ${ }^{1} \mathrm{SM}$ has been hypothesized to be a progressive inflammatory process, moving from mesenteric lipodystrophy to retractile mesenteritis, triggered by a wide variety of stimuli. In this context, similarly to which occurs in atherosclerosis, the change of macrophages resident in mesenteric fat in foamcells related to the upregulation of PPAR- $\gamma$ and scavenger receptor expression might be the starting point in the development of mesenteric panniculitis. ${ }^{14}$ The most common conditions associated with SM are previous abdominal trauma or surgery; ${ }^{8}$ in this context, SM might be the result of an abnormal response to healing and repair of connective tissue after trauma or surgery in genetically predisposed subjects. ${ }^{9}$ Moreover, SM has also been considered a paraneoplastic syndrome, since it is associated with different malignancies, such as lymphomas, chronic lymphocytic leukemia, myeloma, carcinoid, gastrointestinal cancers, renal cancer, and lung cancer. ${ }^{15}$

The rarity of this disease and the lack of clinical trials and practical guidelines have generated many difficulties regarding its definition, the correct establishment of the diagnosis and the possible therapeutic strategies. In 2007, Akram et al reported findings on a sample of 92 patients affected by SM. ${ }^{10}$ After ten years, a systematic review of case reports by Sharma et al identified 192 cases of SM. ${ }^{8}$ Considering these data, SM patients commonly show abdominal pain (75\%), diarrhea/constipation (35\%), palpable abdominal mass $(33.3 \%)$, abdominal tenderness (25\%), weight loss (25\%), nausea and vomiting (20\%), anorexia (15\%), and fever $(12.5 \%) .{ }^{8,10}$ Laboratory findings are non-specific and consist of the increase in inflammatory markers, such as erythrocyte sedimentation rate and C-reactive protein, anemia, leukocytosis, and hypoalbuminemia. ${ }^{8,10,11}$ Regarding the imaging features, the detection at $\mathrm{CT}$ scan of an increase in the mesenteric fat density, named "misty mesentery", should be considered the first indication, but non-specific mark of SM. ${ }^{16}$ Coulier et al described five typical CT scansigns for radiological diagnosis of SM: 1) hyperattenuating mesenteric mass; 2) mass-effect on neighboring mesenteric structures; 3) sub-centimetric nodes in the context of mesenteric fat; 4) "fat halo sign" with the hypoattenuation of fat surrounding the mesenteric nodes or vessels; 5) hyperattenuating pseudo-capsule. ${ }^{17}$ Furthermore, US and magnetic resonance imaging (MRI) have also been performed for the diagnosis of SM, but there are fewer studies for these procedures than $\mathrm{CT}$ and lack of standardized criteria. ${ }^{18-20}$ In this context, Roson et al retrospectively evaluated clinical, $\mathrm{CT}$, and sonographic findings in 26 patients with mesenteric panniculitis, estimating that sonographic features of MS, such as a well-defined homogeneous and hyperechogenic mass, non-deviated vessels within the mass, and displaced bowel loops, well correlated with CT in $92 \%$ of cases. ${ }^{18}$ However, at the moment, although US has been widely used in diagnostic work-up of intestinal diseases characterized by a secondary inflammatory involvement of mesentery (eg, Crohn's disease, colic diverticulitis, peritoneal carcinosis), ${ }^{21-24}$ its use in SM is underestimated and underinvestigated. Given this background, the clinical case we have reported could be a starting point for a wide and standardized use of US in a new clinical scenario.

There is no clear consensus regarding therapeutic strategies, thus the treatment for SM is empirical and varies according to clinical presentation and personal experience. Danford et al proposed a therapeutic algorithm, in which patients are divided into asymptomatic and symptomatic; while the first group are only observed, the second group (the symptomatic group) receive treatment. The first line therapy consists of the use of steroids (prednisone $40 \mathrm{mg} /$ daily tapered slowly) in combination with tamoxifen $10 \mathrm{mg}$ twice daily. ${ }^{9}$ In case of poor improvement of symptoms, the second line therapy to be adopted includes the association between prednisone and azathioprine $2-2.25 \mathrm{mg} / \mathrm{kg}$ daily or simply thalidomide $200 \mathrm{mg}$ daily. ${ }^{9}$ Other medical drugs used successfully, but less frequently, in different case reports are colchicine, ${ }^{25}$ 6-mercaptopurine, ${ }^{26}$ methotrexate, ${ }^{27}$ cyclophosphamide ${ }^{28}$ and infliximab. ${ }^{29}$ Surgery is reserved only in patients with complications, such as bowel obstruction. ${ }^{9,12}$ In our case, the patient was effectively treated using steroids plus tamoxifen.

In conclusion, US could be a sensitive, reliable and non-invasive procedure for the diagnosis and the followup of SM. Multicenter diagnostic trials, including a wide SM population, are needed to define the actual diagnostic accuracy of US in SM.

\section{Ethics Approval and Informed Consent}

This case report has been prepared after obtaining an informed consent from the patient to publish the case details and the accompanying images published. No institutional approval was required to publish the case details. 


\section{Disclosure}

The authors declare that they have no conflicts of interest to disclose.

\section{References}

1. Emory TS, Monihan JM, Carr NJ, Sobin LH. Sclerosing mesenteritis, mesenteric panniculitis and mesenteric lipodystrophy: a single entity? Am J Surg Pathol. 1997;21(4):392-398. doi:10.1097/00000478199704000-00004

2. Jura V. Sulla mesenterite retrattile e sclerosante. Policlinico. 1924;31:575-581.

3. Crane JT, Aguilar MJ, Grimes OF. Isolated lipodystrophy, a form of mesenteric tumor. Am J Surg. 1955;90(2):169-179. doi:10.1016/ 0002-9610(55)90748-0

4. Ogden WW, Bradburn DM, Rives JD. Panniculitis of the mesentery. Ann Surg. 1960;151(5):659-668. doi:10.1097/00000658-19600500000006

5. Yannopoulos K, Stout AP. Primary solid tumors of the mesentery. Cancer. 1963;16:914-927. doi:10.1002/1097-0142(196307) 16:7<914::AID-CNCR2820160708>3.0.CO;2-X

6. Gold RE, Redman HC. Mesenteric fibrosis simulating the angiographic appearance of ileal carcinoid tumor. Radiology. 1972;103 (1):85-86. doi:10.1148/103.1.85

7. Daskalogiannaki M, Voloudaki A, Prassopoulos P, et al. CT evaluation of mesenteric panniculitis: prevalence and associated diseases. AJR Am J Roentgenol. 2000;174(2):427-431. doi:10.2214/ ajr.174.2.1740427

8. Sharma P, Yadav S, Needham CM, Feuerstadt P. Sclerosing mesenteritis: a systematic review of 192 cases. Clin J Gastroenterol. 2017;10(2):103-111. doi:10.1007/s12328-017-0716-5

9. Danford CJ, Lin SC, Wolf JL. Sclerosing mesenteritis. Am J Gastroenterol. 2019;114(6):867-873. doi:10.14309/ ajg.0000000000000167

10. Akram S, Pardi DS, Schaffner JA, Smyrk TC. Sclerosing mesenteritis: clinical features, treatment, and outcome in ninety-two patients. Clin Gastroenterol Hepatol. 2007;May(5):589-596. doi:10.1016/j. cgh.2007.02.032

11. Rispo A, Sica M, Bucci L, et al. Protein-loosing enteropathy in sclerosing mesenteritis. Eur Rev Med Pharmacol Sci. 2015;19 (3):477-480.

12. Yelamanchi R, Yadav E, Gupta N, Durga CK. Idiopathic retractile calcific mesenteritis: a rare cause of small bowel obstruction. Indian J Surg. 2020. doi:10.1007/s12262-020-02537-9

13. Coulier B. Mesenteric panniculitis. Part 1: MDCT pictorial review. JBR-BTR. 2011;94(5):229-240. doi:10.5334/jbr-btr.658

14. Schäffler A, Schölmerich J, Büchler C. Mechanisms of disease: adipocytokines and visceral adipose tissue-emerging role in intestinal and mesenteric diseases. Nat Clin Pract Gastroenterol Hepatol. 2005;2:103-111. doi:10.1038/ncpgasthep0090
15. Vlachos K, Archontovasilis F, Falidas E, Mathioulakis S, Konstandoudakis S, Villias C. Sclerosing mesenteritis: diverse clinical presentations and dissimilar treatment options. A case series and review of the literature. Int Arch Med. 2011;4:17. doi:10.1186/17557682-4-17

16. Mindelzun RE, Jeffrey RB, Lane MJ, Silverman PM. The misty mesentery on CT: differential diagnosis. AJR Am J Roentgenol. 1996;167(1):61-65. doi:10.2214/ajr.167.1.8659422

17. Coulier B. Mesenteric panniculitis. Part 2: prevalence and natural course: MDCT prospective study. JBR-BTR. 2011;94(5):241-246.

18. Biscaldi E, Romairone E, Rollandi GA. Regarding six cases of mesenteric panniculitis: US, spiral CT, magnetic resonance. Radiol Med. 2002;103(5-6):511-518.

19. Rosón N, Garriga V, Cuadrado M, et al. Sonographic findings of mesenteric panniculitis: correlation with $\mathrm{CT}$ and literature review. J Clin Ultrasound. 2006;34(4):169-176. doi:10.1002/jcu.20214

20. Kronthal AJ, Kang YS, Fishman EK, Jones B, Kuhlman JE, Tempany CM. MR imaging in sclerosing mesenteritis. AJR Am J Roentgenol. 1991;156(3):517-519. doi:10.2214/ajr.156.3.1899747

21. Castiglione F, Mainenti PP, De Palma GD, et al. Non-invasive diagnosis of small bowel Crohn's disease: direct comparison of bowel sonography and magnetic resonance enterography. Inflamm Bowel Dis. 2013;19(5):991-998. doi:10.1097/MIB.0b013e3182802b87

22. Maconi G, Greco S, Duca P, et al. Prevalence and clinical significance of sonographic evidence of mesenteric fat alterations in Crohn's disease. Inflamm Bowel Dis. 2008;14(11):1555-1561. doi:10.1002/ibd.20515

23. Lameris W, van Randen A, Bipat S, Bossuyt PM, Boermeester MA, Stoker J. Graded compression ultrasonography and computed tomography in acute colonic diverticulitis: meta-analysis of test accuracy. Eur Radiol. 2008;18(11):2498-2511. doi:10.1007/s00330-008-1018-6

24. Dietrich CF, Hollerweger A, Dirks $K$, et al. EFSUMB Gastrointestinal Ultrasound (GIUS) Task Force Group: celiac sprue and other rare gastrointestinal diseases ultrasound features. Med Ultrason. 2019;21(3):299-315. doi:10.11152/mu-2162

25. Fasoulas K, Beltsis A, Katsinelos T, et al. Efficacy of colchicine in the treatment of mesenteric panniculitis in a young patient. Saudi J Gastroenterol. 2012;18(2):146-148. doi:10.4103/1319-3767.93825

26. Allen PB, De Cruz P, Efthymiou M, Fox A, Taylor AC, Desmond PV. An interesting case of recurrent small bowel obstruction. Case Rep Gastroenterol. 2009;3(3):408-413. doi:10.1159/000254708

27. Viswanathan V, Murray KJ. Idiopathic sclerosing mesenteritis in paediatrics: report of a successfully treated case and a review of literature. Pediatr Rheumatol Online J. 2010;8:5. doi:10.1186/15460096-8-5

28. Bush RW, Hammar SP, Rudolph RH. Sclerosing mesenteritis: response to cyclophosphamide. Arch Intern Med. 1986;146 (3):503-505. doi:10.1001/archinte.1986.00360150113013

29. Rothlein LR, Shaheen AW, Vavalle JP, et al. Sclerosing mesenteritis successfully treated with a TNF antagonist. BMJ Case Rep. 2010;2010:bcr0720103145. doi:10.1136/bcr.07.2010.3145
International Medical Case Reports Journal

\section{Publish your work in this journal}

The International Medical Case Reports Journal is an international, peer-reviewed open-access journal publishing original case reports from all medical specialties. Previously unpublished medical posters are also accepted relating to any area of clinical or preclinical science. Submissions should not normally exceed 2,000 words or 4 published pages including figures, diagrams and references. The manuscript management system is completely online and includes a very quick and fair peer-review system, which is all easy to use. Visit http://www.dovepress.com/testimonials.php to read real quotes from published authors 